\title{
Erratum: Size Tunability and Optical Properties of CdSe Quantum Dots for Various Growth Conditions
}

\author{
[J. Korean Phys. Soc. 62, 121 (2013)]
}

Eun Yee Ko

Center for Nano-Imaging Technology, Korea Research Institute of Standards and Science, Daejeon 305-340, Korea and School of Advanced Materials Engineering and Research Center of Advanced Materials Development, Chonbuk National University, Jeonju 561-756, Korea

Joo In LEE*

Center for Nano-Imaging Technology, Korea Research Institute of Standards and Science, Daejeon 305-340, Korea

Ju-Won JEON and In Hwan $\mathrm{LEE}^{\dagger}$

School of Advanced Materials Engineering and Research Center of Advanced Materials Development, Chonbuk National University, Jeonju 561-756, Korea

Yong Hyeon SHIN

Center for Vacuum Technology, Korea Research Institute of Standards and Science, Daejeon 305-340, Korea

Il $\mathrm{Ki}$ HAN

Center for Opto-Electronic Convergence Systems, Korea Institute of Science and Technology, Seoul 136-791, Korea

DOI: $10.3938 /$ jkps.62.1358

In the acknowledgments, "This research was supported by the Converging Research Center Program through the Ministry of Education, Science and Technology (MEST, 2011K000767) and the National Research Foundation of Korea (NRF) funded by the Korea government (MEST, No. 2010-616- D00056)" should be corrected to "This research was supported by the Converging Research Center Program through the Ministry of Education, Science and Technology (MEST, 2011K000767) and the National Research Foundation of Korea (NRF) funded by the Korea government (MEST, No. 2010-0026614)".

\footnotetext{
*E-mail: jilee@kriss.re.kr; Fax: +82-42-868-5047
}

${ }^{\dagger}$ E-mail: ihlee@jbnu.ac.kr 